\title{
Effect of exciton transfer on recombination dynamics in vertically nonuniform GaAsSb epilayers
}

Bin Zhang, Weiyang Qiu, Shula Chen, Pingping Chen, Weimin Chen, Irina Buyanova and Xingjun Wang

The self-archived postprint version of this journal article is available at Linköping University Institutional Repository (DiVA):

http://urn.kb.se/resolve?urn=urn:nbn:se:liu:diva-159162

N.B.: When citing this work, cite the original publication.

Zhang, B., Qiu, W., Chen, S., Chen, P., Chen, W., Buyanova, I., Wang, X., (2019), Effect of exciton transfer on recombination dynamics in vertically nonuniform GaAsSb epilayers, Applied Physics Letters, 114(25), 252101. https://doi.org/10.1063/1.5105343

Original publication available at:

https://doi.org/10.1063/1.5105.343

Copyright: AIP Publishing

http://www.aip.org/ 


\title{
Effect of exciton transfer on recombination dynamics in vertically nonuniform GaAsSb epilayers
}

\section{Bin Zhang1, ${ }^{1,}$ Weiyang Qiu${ }^{1}$, Shula Chen ${ }^{2}$, Pingping Chen ${ }^{1}$, Weimin M Chen ${ }^{2}$, Irina A. Buyanova $^{2, a)}$ and Xingjun Wang ${ }^{1, b)}$}

${ }^{1}$ State key Laboratory for Infrared Physics, Shanghai Institute of Technical Physics, Chinese Academy of Sciences, Shanghai, 200083, China

${ }^{2}$ Department of Physics, Chemistry and Biology, Linköping University, S-581 83 Linköping, Sweden

${ }^{3}$ University of Chinese Academy of Sciences, Beijing 100049, China

a) Email: iribu@ifm.liu.se

b) Email: xjwang@mail.sitp.ac.cn

\begin{abstract}
Low-temperature photoluminescence (PL), photoreflectance (PR), and temperature dependent time-resolved PL spectroscopies are employed to investigate optical emission processes and exciton dynamics in graded GaAsSb epilayers. The nonuniformity in the $\mathrm{Sb}$ composition along the growth direction is disclosed from low-temperature PL and PR measurements. Furthermore, significant differences in PL dynamics are found at low temperatures for the PL emissions originating from spatial regions with the low and high $\mathrm{Sb}$ compositions, with a fast decay and a slow rise at the early stage of the PL transient, respectively. This finding is attributed to exciton transfer from the low $\mathrm{Sb}$ region to the high $\mathrm{Sb}$ region. The obtained results are important for a general understanding of optical transitions and exciton/carrier dynamics in material systems with graded alloy composition.
\end{abstract}

PACS numbers: 78.30.Fs, 78.47.+p, 78.55.-m, 78.55.Cr 
The bandgap of GaAsSb encompasses the important wavelength range from $\sim 0.87 \mu \mathrm{m}(\mathrm{GaAs})$ to $\sim 1.7 \mu \mathrm{m}$ (GaSb). ${ }^{1,2}$ This makes GaAsSb suitable for a variety of potential optoelectronic applications: infrared light-emitting diodes,${ }^{3}$ photodetectors, ${ }^{4}$ terahertz quantum cascade lasers ${ }^{5}$ and optical telecommunications operating at $\sim 1.55 \mu \mathrm{m} .{ }^{6}$ Furthermore, an increase in spin-orbit interaction due to introduction of heavy $\mathrm{Sb}$ atoms into GaAs induces sub-picosecond spin relaxation. This makes GaAsSb an ideal material for future ultrafast all-optical polarization switches at room temperature. ${ }^{7,8}$ Most recently, a high electron spin polarization up to $\sim 21 \%$ was observed in $\mathrm{GaAsSb}$ with $\mathrm{Sb}$ composition of $\sim 6 \%$, which raises the prospect of realizing future scalable spintronic devices based on GaAs and related material systems. ${ }^{9}$

However, in spite of great application potential of GaAsSb alloys, it has been found that accurate compositional control in such system is still difficult. In contrast to conventional IIIIII'-V alloy systems such as AlGaAs, strong competition between two group-V elements in GaAsSb leads to their non-unity sticking coefficients, which complicates the incorporation behavior of $\mathrm{Sb}$ and As atoms. ${ }^{10,11}$ It was shown that a naturally formed Sb gradients exists in GaAsSb epitaxial layers grown by molecular beam epitaxy (MBE). ${ }^{12}$ Moreover, a consistent self-induced Sb compositional gradients are also observed in GaAsSb nanowires grown by MBE, leading to a specific and reproducible rectifying behavior. ${ }^{13}$ It means that the compositional non-uniformity in semiconductor alloys may strongly affect their optical and electrical properties. ${ }^{13-15}$ To date, however, a comprehensive understanding of optical transitions and exciton/carrier dynamics in such material systems is lacking. Hence, in this work, the effects of compositional non-uniformity in GaAsSb layers on radiative recombination processes are characterized in detail by photoreflectance $(\mathrm{PR})$ and temperature dependent timeresolved photoluminescence (PL) spectroscopies. At low temperatures, photo-excited carriers are found to be localized within two regions of the GaAsSb layer with different Sb contents, which has a profound effect on dynamics of radiative recombination investigated by using 
transient PL (TRPL) measurements. The finding is attributed to transfer of photo-generated excitons between the regions with different $\mathrm{Sb}$ compositions, as confirmed by temperature dependent TRPL studies.

The GaAsSb epilayers were grown on semi-insulating GaAs (001) substrates by molecular beam epitaxy at $530^{\circ} \mathrm{C}$. The growth process continued for 105 minutes with the deposition rate of $10 \mathrm{~nm} / \mathrm{min}$. The rather high growth temperature chosen here is expected to lead to graded $\mathrm{Sb}$ composition along the growth direction due to phase separation. ${ }^{16}$ For PR and PL measurements, the solid-state 405-nm and 785-nm lasers were employed for optical pumping of the sample. The probe beam during the PR measurements was provided by a broadband white light source (Laser-Driven Light Source). ${ }^{17}$ The PL and PR signals were collected in a strict backscattering geometry and analyzed by a Tri-Vista spectrometer equipped with a Si Avalanche Photo Diode (APD) and a cooled InGaAs detector. Finally, the modulated signals were amplified by a SR 830 Lock-in Amplifier at the modulation frequency of the pumping beam. Time-resolved PL measurements were conducted by employing as an excitation light source a mode-locked picosecond Ti:Sapphire laser with a repetition frequency of $76 \mathrm{MHz}$ and a temporal resolution of $1.5 \mathrm{ps}$. The excitation wavelength was set at $760 \mathrm{~nm}$. Transient PL signals were detected by a streak camera system.

Typical low-temperature ( $\mathrm{T}=13 \mathrm{~K})$ PR and PL spectra of the GaAsSb epilayer under the 405nm excitation are shown in Fig. 1(a). The PR spectrum contains a single feature labeled as El. The corresponding energy, denoted by the vertical arrow in Fig. 1(a), was determined by fitting the experimental data using the Aspnes' third derivate function. ${ }^{18}$ Since PR spectra are insensitive to localized states, this energy corresponds to the fundamental bandgap of the GaAsSb alloy. ${ }^{19}$ We also observe that the PL peak position lies below El, which means that the PL emission exhibits a large Stokes shift of $\sim 20 \mathrm{meV}$. This suggests that the PL emission is due to recombination of localized excitons (LEs) trapped by random potential fluctuations. Below 
we will strengthen this point by providing additional experimental evidence.

When the excitation light is tuned to a longer wavelength, i.e. $785 \mathrm{~nm}$ in this case, a new feature $E_{h}$ appears at the lower energy side of the PR spectrum, as shown in Fig. 1(b). To understand its origin, we recall that PR spectra reflect the energy band structure within the spatial region defined by the penetration depth of the excitation light. Given the fact that incorporating a small amount of $\mathrm{Sb}$ atoms into GaAs has no substantial influence on the absorption coefficients for photon energies exceeding energies of optical transitions involving the spin-orbit-split valence subband, the penetration depth of the $405 \mathrm{~nm}$ light in the investigated sample can be estimated as $\sim 15 \mathrm{~nm} .{ }^{15,20}$ Thus the high-energy feature $E_{1}$ in Fig. 1 (a-b) stems from the optical transitions in the near-surface region with a lower Sb content. Since the 785-nm excitation light has a longer penetration depth of $\sim 0.7 \mu \mathrm{m}$ in $\mathrm{GaAs}^{21}$, the observed lower-energy transition should originate from the region farther away from the surface. This means that the Sb composition is slightly higher in the deeper bulk region. This conclusion is supported by our secondary-ion mass spectrometry (SIMS) measurements, which show that the $\mathrm{Sb}$ composition is lower within first $150 \mathrm{~nm}$ from the surface (see Supplementary Material). The observation of the Eh transition is accompanied by the appearance of a new PL band, which peaks at $1.304 \mathrm{eV}$. This emission also exhibits a significant Stokes shift of about $25 \mathrm{meV}$ relative to the $E_{h}$ energy, which again suggests that the excitons involved in the corresponding radiative recombination may experience localization.

Indeed, excitons in semiconductor alloys are often confined in potential minima caused by compositional fluctuations. According to the previous studies ${ }^{22,23}$, increasing the excitation power (P) will lead to gradual filling of the localized states. Correspondingly, PL spectra related to localized exciton recombination typically exhibit a blue shift with increasing $\mathrm{P}$. To confirm that the detected PL emissions are indeed of the LE origin, we have measured low-temperature PL spectra under photo-excitation at $785 \mathrm{~nm}$ with different excitation powers and the 
corresponding results are displayed in Fig. 2. The dramatic blue shift of the high-energy emission peak from $1.359 \mathrm{eV}$ to $1.378 \mathrm{eV}$ was observed, providing a direct experimental proof for the exciton localization. Moreover, the low-energy emission band emerges at $\mathrm{P}=200 \mathrm{P}_{0}$ and shifts to higher energies with increasing excitation power. (Here $\mathrm{P}_{0}=2.9 \mathrm{~mW} / \mathrm{cm}^{2}$ denotes the minimum excitation power used during the measurements). This is accompanied by a dramatic increase in the intensity of this PL band, which dominates the PL spectra measured at the high P. Such power dependence can be explained assuming exciton transfer between the regions with different Sb compositions. At low excitation powers, the PL is dominated by the high-energy PL band as the number of the photo-generated carriers is the highest within the near-surface region with the low $\mathrm{Sb}$ content. With increasing $\mathrm{P}$, the photo-generated excitons gradually fill the localized states which leads to the observed gradual blue-shift of the high-energy PL band (see Fig. 2). This facilitates the exciton transfer from the low-Sb region to the high-Sb region. Therefore, at an intermediate excitation power (here $\mathrm{P}=200 \mathrm{P}_{0}$ ), the low-energy emission band starts to emerge in the PL spectra. Under the highest P, the localized states in the low-Sb region become almost saturated and the excitons are freely transferred to the high-Sb region leading to the dominance of the low-energy PL band.

In order to gain further information on the origin of the detected PL emissions, dynamics of the corresponding radiative transitions was studied by employing time-resolved PL spectroscopy. The streak image of the time resolved PL emission intensity as a function of photon energy measured at $\mathrm{T}=10 \mathrm{~K}$ from the investigated sample is shown in Fig. 3(a). It is noticeable that two PL bands exhibit different temporal behavior: the PL spectrum is initially dominated by the high-energy band immediately after the excitation pulse, whereas the lowenergy PL band prevails when the delay time exceeds 1140 ps. These differences in the PL dynamics are further visualized in Fig. 3(b) and (c), where temporally-resolved PL spectra extracted from the original image within various temporal intervals after the excitation pulse 
and temporal profiles of the PL emission at different detection energies are shown, respectively.

Firstly, the high-energy PL band reaches its peak intensity within first 140 ps, whereas a significantly slower rising of 714 ps is observed for the low-energy emission band. This can also be seen from Fig. 3(b), where temporally-resolved PL spectra extracted from the original image within various temporal intervals after the excitation pulse are shown. Secondly, the maximum position of the high-energy PL band (marked by the magenta circles in Fig.3(b)) continuously shifts towards lower photon energies as time elapses. In contrast, the low-energy emission shifts to higher energies within the first $714 \mathrm{ps,} \mathrm{which} \mathrm{is} \mathrm{followed} \mathrm{by} \mathrm{the} \mathrm{red} \mathrm{shift} \mathrm{with}$ the further time delay $\left(\Delta \mathrm{t}_{\mathrm{d}}\right)$ after the excitation pulse. Consequently, the transient PL spectrum is dominated initially by the high-energy emission band just after the pulsed excitation and finally by the lower-energy emission band as the high energy one rapidly quenches.

The red shift of the high-energy PL band with increasing $\Delta \mathrm{t}_{\mathrm{d}}$ can be explained within the framework of localized exciton recombination, i.e. it can be attributed to the transfer of the localized excitons from shallow into deeper localized states with time. Similar transient behavior of the LE emission has also been observed in other alloy systems, such as GaNAs ${ }^{24}$, $\mathrm{InGaN}^{25,26}$ and $\mathrm{ZnCdO}^{27}$. The red shift of the low-energy emission band, which follows the slow increase of its intensity at the initial stage of the PL transient, also implies its LE nature, consistent with the photoreflectance and cw-PL results discussed above. On the other hand, the initial blue shift and the accompanying slow rising process seems to be unusual.

To provide further insight into the time evolution of the monitored radiative transitions, the PL transients were monitored at various energy positions within the PL spectra - Fig. 3(c). The intensity of each curve is adjusted by multiplying it with a certain constant, for easier comparison. We note that the PL decay profile of the high-energy band is characterized by a double exponential function, which suggests that it involves two decay components with the fast one dominating at the beginning of the PL decay. On the other hand, the low-energy 
emission band exhibits a slow rise stage followed by a slow PL decay. We attribute this to exciton transfer from the low $\mathrm{Sb}$ content region to the high $\mathrm{Sb}$ content region, so that the fast PL decay of the high-energy PL band and the slow rising process of the low-energy PL band correspond to exciton escape and injection processes, respectively.

As shown in Fig. 3 (b) and (c), this exciton transfer has a non-negligible influence on the radiative recombination and, therefore, needs to be included in the analysis of its dynamics. Considering that the characteristic thermal energy $(\sim \mathrm{kBT})$ is far less than the barrier energy between the two regions ( $57 \mathrm{meV}$ ) determined from the PR measurements (see Fig.1), a reverse thermal escape of excitons from the high $\mathrm{Sb}$ content region is negligible in our case. Furthermore, the efficiency of exciton transfer is expected to decrease with increasing distance between the localized states. ${ }^{28}$ For example, according to the previous studies ${ }^{29}$, energy transfer of excitons localized in a GaAs core far from the core/cell interface was found to be inefficient at low temperatures, leading to a weak enhancement in PL excitation (PLE) intensity for the GaAsN cell emission. Based on the above analysis, we developed the following simplified set of rate equations with a minimal number of fitting parameters to visualize the role of exciton transfer:

$$
\begin{aligned}
& \frac{d n_{l 1}}{d t}=-\frac{n_{l 1}}{\tau_{l}}, \\
& \frac{d n_{l 2}}{d t}=-\frac{n_{l 2}}{\tau_{l}}-\frac{n_{l 2}}{\tau_{\text {tran }}} \\
& \frac{d n_{h 2}}{d t}=-\frac{n_{h 2}}{\tau_{h}}+\frac{n_{l 2}}{\tau_{\text {tran }}}, \\
& \frac{d n_{h 1}}{d t}=-\frac{n_{h 1}}{\tau_{h}},
\end{aligned}
$$

where $\mathrm{n}_{l 1}\left(\mathrm{n}_{l 2}\right)$ and $\mathrm{n}_{h 1}\left(\mathrm{n}_{h 2}\right)$ are the exciton densities in the low and high Sb content regions respectively. The subscript $1(2)$ denotes the excitons trapped by the localized states that are far from (close to) the interface between these regions. $\tau_{\text {tran }}$ is the characteristic time of the exciton transfer from the low $\mathrm{Sb}$ content region into the high $\mathrm{Sb}$ content region, and $\tau_{l}\left(\tau_{h}\right)$ is the 
effective recombination lifetime in the low (high) Sb content region. Eqs. (1) and (4) describe recombination dynamics of the excitons, which are localized far away from the interface between the regions, and thus do not efficiently contribute to the exciton transfer, whereas Eqs. (2) and (3) include the additional exciton transfer. A simplified sketch of these processes is shown in Fig. 4(a).

At low temperatures, the exciton transfer is limited by their localization at the band tail states caused by alloy fluctuations. According to our previous studies ${ }^{9}$, increasing temperature leads to gradual thermal delocalization of the trapped excitons. It is thus expected that the influence of exciton trapping on the transfer process becomes less significant at elevated temperatures. To gain further understanding of the exciton transfer, temperature dependent TRPL measurements were carried out on the investigated sample. The temporal profiles detected at various temperatures at the PL maximum energies of the high-energy and low-energy PL bands are shown by the symbols in Fig. 4(b) and (c), respectively. The corresponding simulation curves using the rate equation analysis are displayed in the figure by the solid lines. From the best fit to the experimental data, the characteristic transfer time $\tau_{\text {tran }}$ and effective exciton lifetime $\tau_{l}\left(\tau_{h}\right)$ can then be extracted as depicted in Fig. 4(d). The exciton transfer time is found to gradually decrease with increasing temperature, which is also reflected by the shortened 'fast decay' and 'slow rise' stages for the high- and low-energy PL components in Fig. 4(b) and (c), respectively. Interestingly, $\tau_{\text {tran }}$ changes only slightly at $\mathrm{T}>110 \mathrm{~K}$, which indicates that exciton/carriers at such temperatures have enough thermal energy to escape from the localization potential and become fully delocalized. This is further supported by TRPL measurements - see Supplementary Material.

Furthermore, we observed that the effective exciton lifetime is longer in the high $\mathrm{Sb}$ content region than in the low $\mathrm{Sb}$ region, and that both of them rapidly decline with increasing temperature, as depicted in Fig. 4(d). The effective exciton lifetime $(\tau)$ is determined by the 
competition between radiative $\left(\tau_{r}\right)$ and nonradiative $\left(\tau_{n r}\right)$ lifetimes, as described by $1 / \tau=$ $1 / \tau_{r}+1 / \tau_{n r}$. At low temperatures, excitons are trapped at the localization states, which suppresses their capture by competing nonradiative recombination centers. $\tau_{l}$ is found to be shorter than $\tau_{h}$, which may be attributed to the combined effects of decreased exciton localization in the low $\mathrm{Sb}$ region and also its proximity to the surface. At elevated temperatures, however, the localized excitons become thermally activated into extended states and the exciton lifetime is dominated by nonradiative recombination. This leads to a rapid decrease of the PL decay time observed experimentally.

In summary, we have investigated effects of compositional nonuniformity in the GaAsSb epilayers on the radiative recombination and its dynamics by using temperature-dependent TRPL measurements complemented by the rate equation analysis. This compositional nonuniformity was revealed from the performed PL and PR measurements, which showed that the $\mathrm{Sb}$ content is lower near the surface but increases in the regions farther away from the surface. Furthermore, the LE emissions from the areas with the low and high Sb content are found to exhibit significantly different dynamics at low temperatures. The temporal profiles of the LE emission from the low $\mathrm{Sb}$ content region exhibits a fast decay at the beginning stage of the PL transient which is accompanied by a slow rise of the LE component from the high $\mathrm{Sb}$ content region. This finding suggests that the exciton transfer process from the low Sb region into the high $\mathrm{Sb}$ region should be included into the exciton lifetime analysis. The semiquantitative rate equation model is developed to investigate the temperature dependence of the exciton dynamics. The results indicate that the localization potential in the low $\mathrm{Sb}$ region is the main factor limiting exciton transfer at low temperatures. We note that the provided analysis should be applicable to other distributions of the alloy composition including spinodal decomposition of the alloy. 


\section{Supplementary Material.}

See supplementary material for PL dynamics at $\mathrm{T}=80 \mathrm{~K}$ and $110 \mathrm{~K}$, and for the SIMS depth profile of the GaAsSb epilayer.

Acknowledgement. This work is supported by the National Natural Science Foundation of China (Grant Nos. 61274141 and 11874377), Shanghai Science and Technology Foundation (Grant No. 18JC1420401) and Natural Science Foundation of Shanghai (Grant No. 18ZR1445700). B. Zhang acknowledges the support from China Scholarship Council (No. 201604910582). 


\section{References}

1. R. Lukic-Zrnic, B. P. Gorman, R. J. Cottier, T. D. Golding, C. L. Littler and A. G. Norman, Journal of Applied Physics 92 (11), 6939 (2002).

2. R. M. Cohen, M. J. Cherng, R. E. Benner and G. B. Stringfellow, Journal of Applied Physics 57 (10), 4817-4819 (1985).

3. R. B. Lewis, D. A. Beaton, X. F. Lu and T. Tiedje, J. Crystal. Growth 311 (7), $1872-$ 1875 (2009).

4. X. Sun, S. Wang, J. S. Hsu, R. Sidhu, X. G. Zheng, X. Li, J. C. Campbell and J. AL Holmes, IEEE JOURNAL OF SELECTED TOPICS IN QUANTUM ELECTRONICS 8 (4), 817-822 (2002).

5. C. Deutsch, M. Krall, M. Brandstetter, H. Detz, A. M. Andrews, P. Klang, W. Schrenk, G. Strasser and K. Unterrainer, Appl. Phys. Lett 101 (21), 211117 (2012).

6. E. Ahmad, M. R. Karim, S. Bin Hafiz, C. L. Reynolds, Y. Liu and S. Iyer, Sci Rep-Uk 7, 12 (2017).

7. M. Motyka, F. Janiak, G. Sęk, J. Misiewicz and K. D. Moiseev, Applied Physics Letters 100 (21), 211906 (2012).

8. K. Hall, S. Leonard, H. van Driel, A. Kost, E. Selvig and D. Chow, Applied Physics Letters 75 (23), 3665-3667 (1999).

9. W. Qiu, X. Wang, P. Chen, N. Li and W. Lu, Applied Physics Letters 105 (8), 082104 (2014).

10. H. P. Hsu, P. Sitarek, Y. S. Huang, P. W. Liu, J. M. Lin, H. H. Lin and K. K. Tiong, J Phys Condens Matter 18 (26), 5927-5935 (2006).

11. M. Yano, Journal of Vacuum Science \& Technology B: Microelectronics and Nanometer Structures 7 (2), 199 (1989).

12. Y. M. Lin, C. H. Chen, J. S. Wu and C. P. Lee, Journal of Crystal Growth 402, 151-154 
(2014).

13. J. Huh, H. Yun, D. C. Kim, A. M. Munshi, D. L. Dheeraj, H. Kauko, A. T. van Helvoort, S. Lee, B. O. Fimland and H. Weman, Nano Lett 15 (6), 3709-3715 (2015).

14. X. Gao, Z. Wei, F. Zhao, Y. Yang, R. Chen, X. Fang, J. Tang, D. Fang, D. Wang, R. Li, X. Ge, X. Ma and X. Wang, Sci Rep 6, 29112 (2016).

15. K. Alberi, J. Wu, W. Walukiewicz, K. M. Yu, O. D. Dubon, S. P. Watkins, C. X. Wang, X. Liu, Y. J. Cho and J. Furdyna, Physical Review B 75 (4) (2007).

16. B.-R. Wu, C. Xu, K.-L. Chang, K.-C. Hsieh and K. Y. Cheng, Journal of Vacuum Science \& Technology B: Microelectronics and Nanometer Structures 23 (4) (2005).

17. B. Zhang and X.-J. Wang, Review of Scientific Instruments 88 (10), 106103 (2017).

18. B. Zhang, W. Y. Qiu, P. P. Chen and X. J. Wang, Journal of Applied Physics 123 (3) (2018).

19. R. Kudrawiec, G. Sęk, J. Misiewicz, F. Ishikawa, A. Trampert and K. H. Ploog, Applied Physics Letters 94 (1), 011907 (2009).

20. D. E. Aspnes and A. A. Studna, Physical Review B 27 (2), 985-1009 (1983).

21. G. Abstreiter, E. Bauser, A. Fischer and K. Ploog, Applied physics 16 (4), 345-352 (1978).

22. B. Zhang, C. Chen, J. B. Han, C. Jin, J. X. Chen and X. J. Wang, Aip Advances 8 (4) (2018).

23. I. A. Buyanova, W. M. Chen, G. Pozina, J. P. Bergman, B. Monemar, H. P. Xin and C. W. Tu, Applied Physics Letters 75 (4), $501-503$ (1999).

24. I. A. Buyanova, G. Pozina, P. N. Hai, N. Q. Thinh, J. P. Bergman, W. M. Chen, H. P. Xin and C. W. Tu, Applied Physics Letters 77 (15), 2325-2327 (2000).

25. Y. Narukawa, Y. Kawakami, S. Fujita, S. Fujita and S. Nakamura, Physical Review B 55 (4), R1938 (1997). 
26. Y. L. Li, X. J. Wang, S. M. He, B. Zhang, L. X. Sun, Y. D. Li, Q. Guo, C. Q. Chen, Z. H. Chen and W. Lu, Journal of Applied Physics 112 (12), 123515 (2012).

27. I. A. Buyanova, J. P. Bergman, G. Pozina, W. M. Chen, S. Rawal, D. P. Norton, S. J. Pearton, A. Osinsky and J. W. Dong, Applied Physics Letters 90 (26), 261907 (2007).

28. S. K. Lyo, Physical Review B 62 (20), 13641-13656 (2000).

29. S. Chen, M. Jansson, S. Filippov, F. Ishikawa, W. M. Chen and I. A. Buyanova, Journal of Vacuum Science \& Technology B, Nanotechnology and Microelectronics: Materials, Processing, Measurement, and Phenomena 34 (4) (2016). 


\section{FIGURE CAPTIONS}

Figure 1. Low-temperature $(\mathrm{T}=13 \mathrm{~K})$ photoreflectance (the red curves) and photoluminescence spectra (the black curves) from the syudied GaAsSb epilayer under the excitation of the 405-

nm laser (a) and the 785-nm laser (b). The vertical arrow mark the critical-point energies determined from the best fit to the PR spectra.

Figure 2. Power dependent PL spectra measured at $10 \mathrm{~K}$ under photo- excitation at $785 \mathrm{~nm}$, with excitation power increasing by a factor of 90000 from $\mathrm{P}_{0}=2.9 \mathrm{~mW} / \mathrm{cm}^{2}$. Each spectrum was normalized to peak intensity of the high-energy PL band and vertically shifted for easier comparison. The open circles (open triangles) mark the peak position of high (low)-energy PL emission band.

Figure 3. (a) Streak image of the time-resolved PL emission from the investigated sample measured at $\mathrm{T}=10 \mathrm{~K}$. (b) Transient PL intensity profiles extracted from the original streak image the the specified delay times after the laser pulse. The low-energy and high-energy PL emission bands are from the high $\mathrm{Sb}$ and low $\mathrm{Sb}$ region, respectively. (c) PL decay curves extracted at different emission energies from the streak image.

Figure 4 (a) Simplified schematic diagram for exciton dynamics in the investigated sample. (b) and (c) Temporal profiles at the peak energy of the PL emissions from the low Sb and high Sb region, respectively. The solid lines are the simulated curves to the experimental data (the open circles), obtained from the rate equation analysis. For easier visualization, the PL decay curves are displaced vertically. (d) The characteristic exciton transfer time (the blue filled triangles) and effective recombination times of the excitons from the low $\mathrm{Sb}$ (the red filled squares) and the high $\mathrm{Sb}$ region (the green filled circles) as a function of temperature. 
Wavelength (nm)

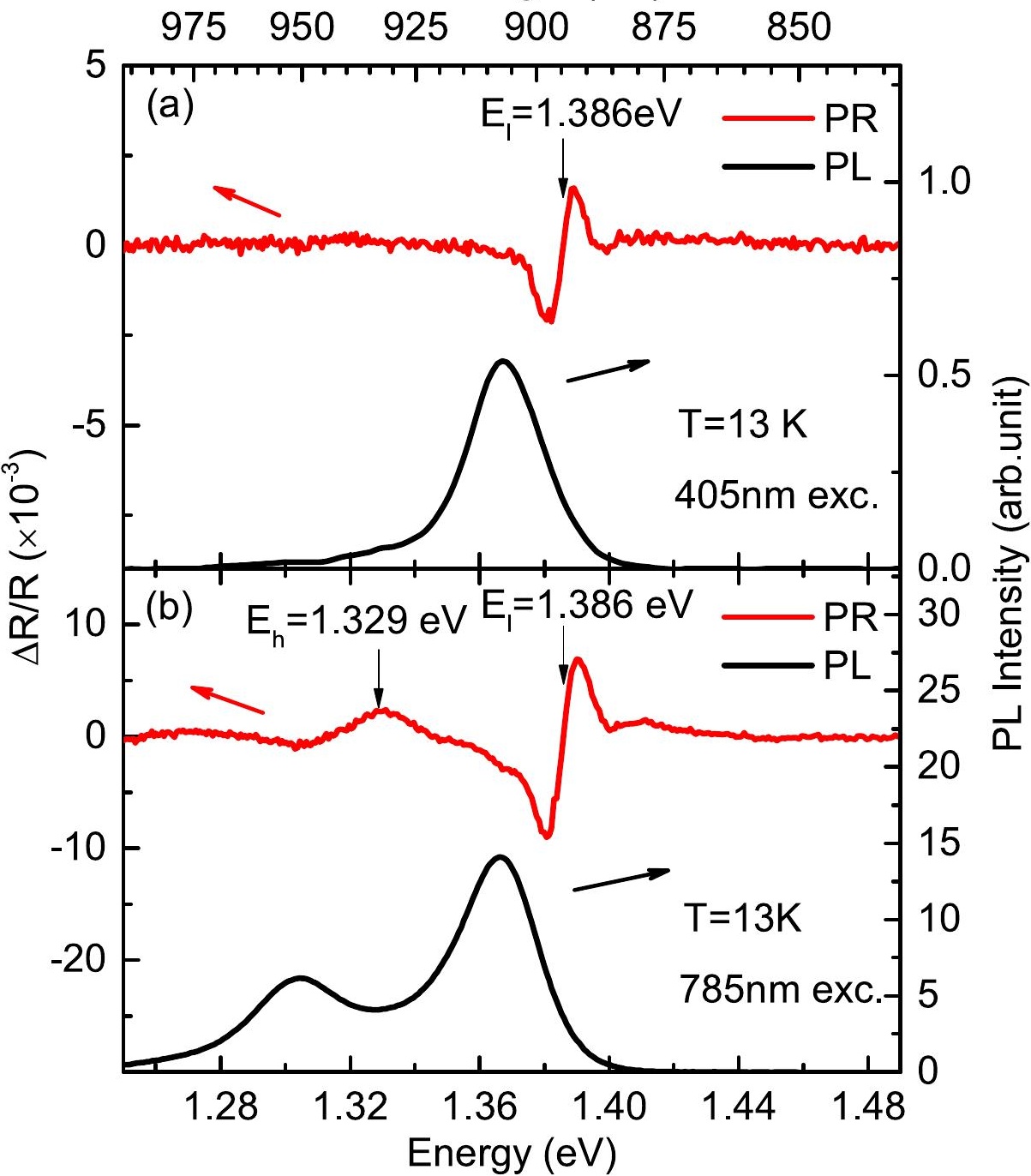




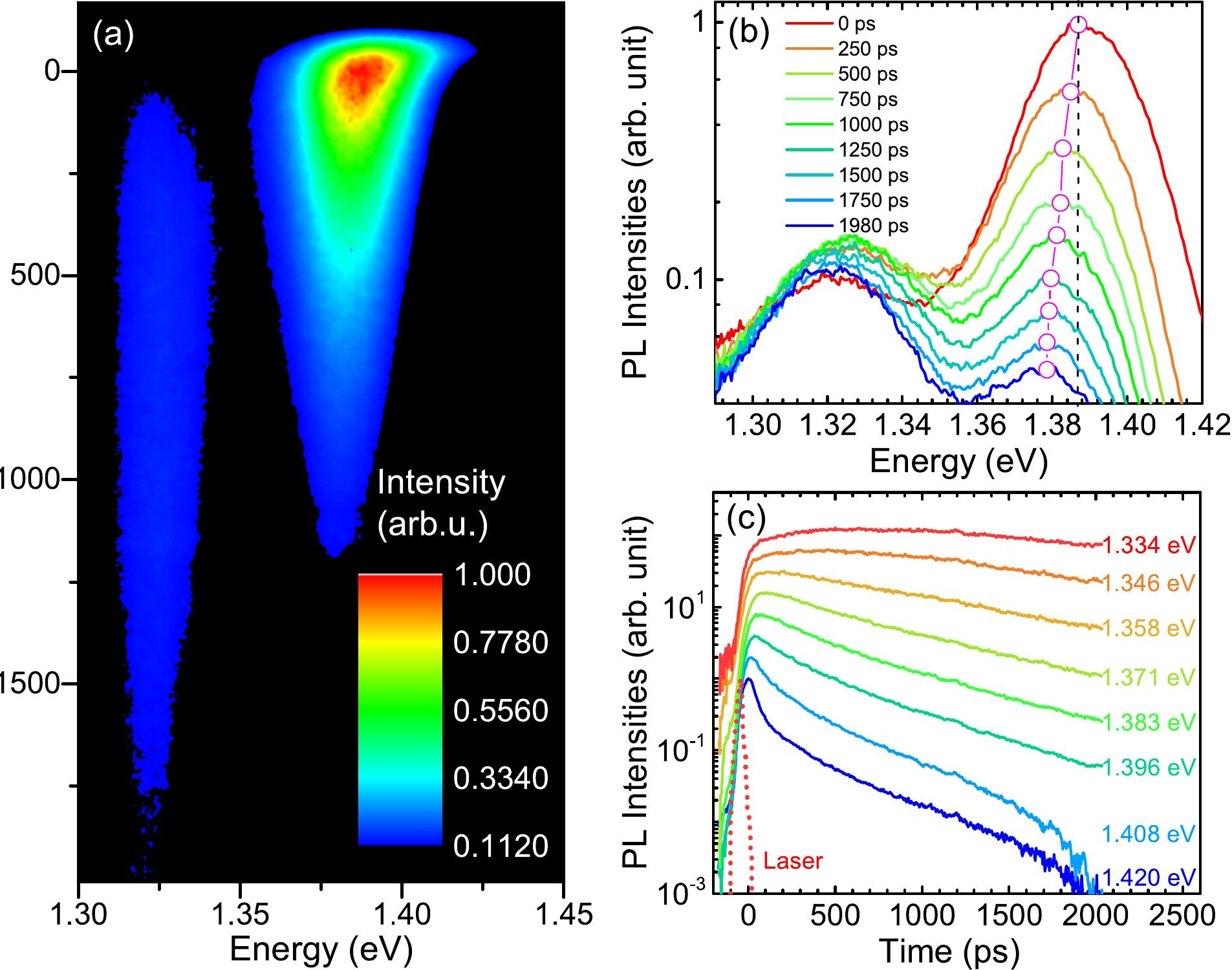



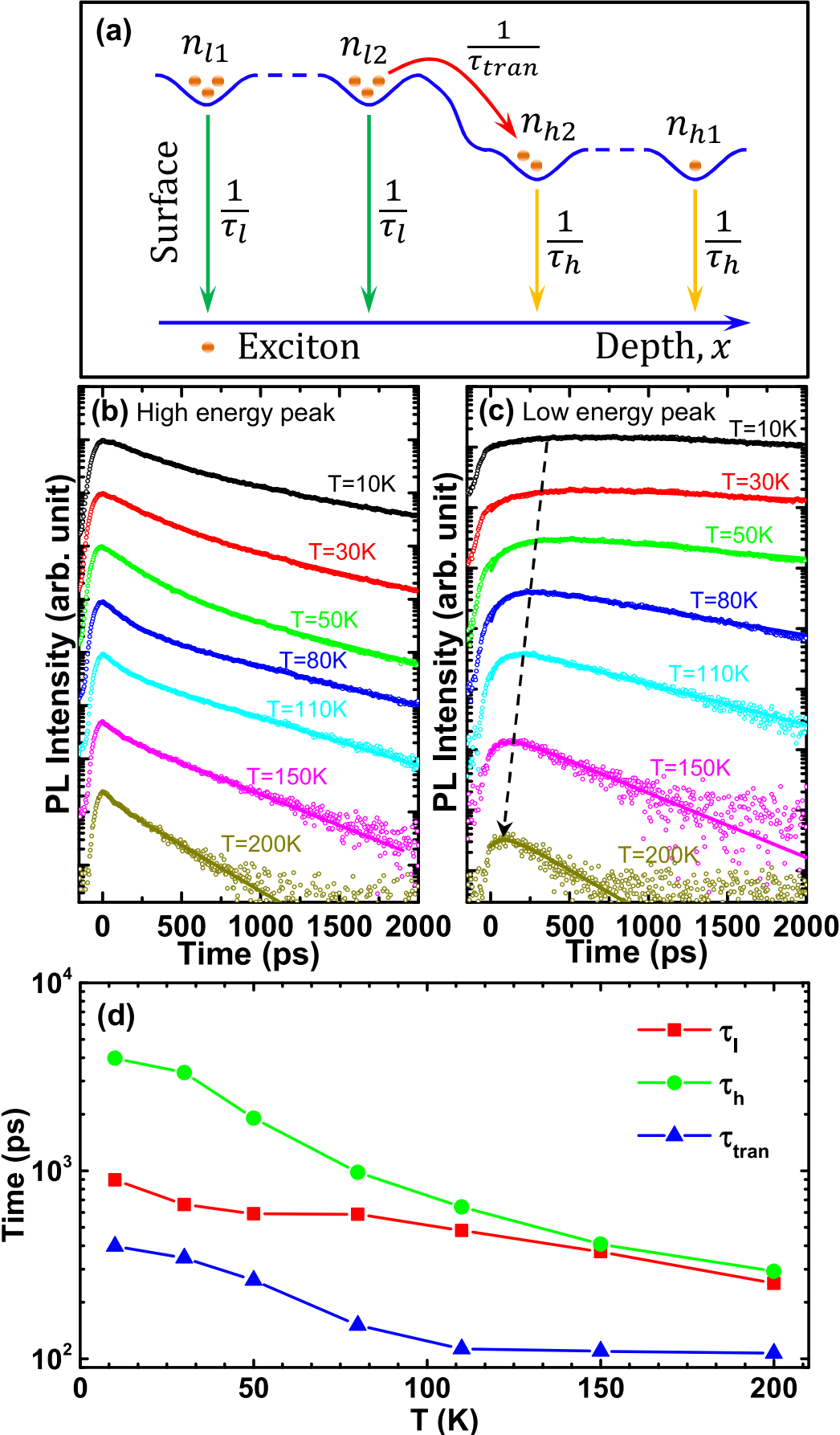